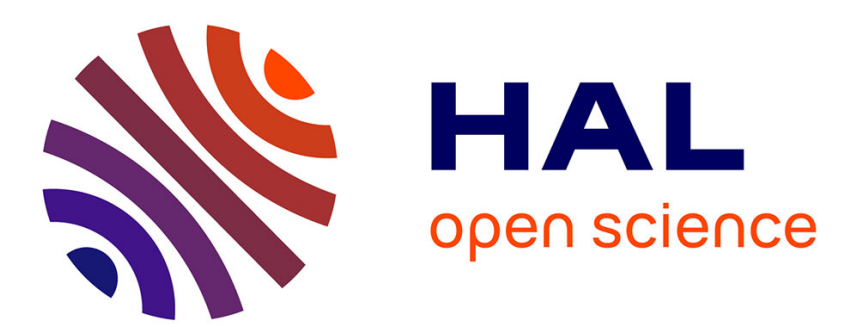

\title{
High-throughput Phenotyping and Genomic Selection: The Frontiers of Crop Breeding Converge
}

Llorenç Cabrera-Bosquet, José Crossa, Jarislav von Zitzewitz, María Dolors Serret, José Luis Araus

\section{- To cite this version:}

Llorenç Cabrera-Bosquet, José Crossa, Jarislav von Zitzewitz, María Dolors Serret, José Luis Araus. High-throughput Phenotyping and Genomic Selection: The Frontiers of Crop Breeding Converge. Journal of Integrative Plant Biology, 2012, 54 (5), pp.312-320. 10.1111/j.1744-7909.2012.01116.x . hal-01001607v2

\section{HAL Id: hal-01001607 \\ https://hal.science/hal-01001607v2}

Submitted on 19 Sep 2019

HAL is a multi-disciplinary open access archive for the deposit and dissemination of scientific research documents, whether they are published or not. The documents may come from teaching and research institutions in France or abroad, or from public or private research centers.
L'archive ouverte pluridisciplinaire $\mathbf{H A L}$, est destinée au dépôt et à la diffusion de documents scientifiques de niveau recherche, publiés ou non, émanant des établissements d'enseignement et de recherche français ou étrangers, des laboratoires publics ou privés. 


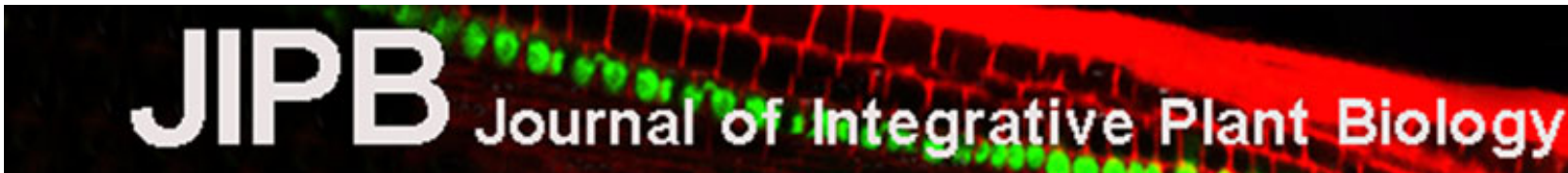

Journal of Integrative Plant Biology 2012, 54 (5): 312-320

\section{Invited Expert Review}

\section{High-throughput Phenotyping and Genomic Selection: The Frontiers of Crop Breeding Converge ${ }^{\boxplus}$}

\author{
Llorenç Cabrera-Bosquet ${ }^{1}$, José Crossa ${ }^{2}$, Jarislav von Zitzewitz ${ }^{3}$, María Dolors Serret ${ }^{4}$ \\ and José Luis Araus ${ }^{4}$ \\ ${ }^{1}$ French National Institute for Agricultural Research (INRA, UMR759), Ecophysiology Laboratory of Plants under Environmental Stress, \\ Montpellier F-34060, France \\ ${ }^{2}$ International Maize and Wheat Improvement Center (CIMMYT), El Batán, Texcoco CP 56130, Mexico \\ ${ }^{3}$ National Research Program on Rainfed Crops, National Institute for Agricultural Research, Est. Exp. La Estanzuela, \\ Colonia 70000, Uruguay \\ ${ }^{4}$ Unit of Plant Physiology, Faculty of Biology, University of Barcelona, Av. Diagonal, 643, Barcelona 08028, Spain \\ "Corresponding author \\ Tel: +34 93402 1469; Fax: +34 93411 2842; E-mail: jaraus@ub.edu \\ F Articles can be viewed online without a subscription. \\ Available online on 16 March 2012 at www.jipb.net and www.wileyonlinelibrary.com/journal/jipb \\ doi: 10.1111/j.1744-7909.2012.01116.x
}

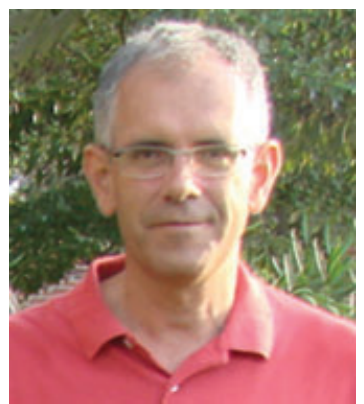

José Luis Araus

(Corresponding author)

\section{Abstract}

Genomic selection (GS) and high-throughput phenotyping have recently been captivating the interest of the crop breeding community from both the public and private sectors world-wide. Both approaches promise to revolutionize the prediction of complex traits, including growth, yield and adaptation to stress. Whereas high-throughput phenotyping may help to improve understanding of crop physiology, most powerful techniques for high-throughput field phenotyping are empirical rather than analytical and comparable to genomic selection. Despite the fact that the two methodological approaches represent the extremes of what is understood as the breeding process (phenotype versus genome), they both consider the targeted traits (e.g. grain yield, growth, phenology, plant adaptation to stress) as a black box instead of dissecting them as a set of secondary traits (i.e. physiological) putatively related to the target trait. Both GS and high-throughput phenotyping have in common their empirical approach enabling breeders to use genome profile or phenotype without understanding the underlying biology. This short review discusses the main aspects of both approaches and focuses on the case of genomic selection of maize flowering traits and near-infrared spectroscopy (NIRS) and plant spectral reflectance as high-throughput field phenotyping methods for complex traits such as crop growth and yield.

Keywords: Genomic selection; high-throughput phenotyping; NIRS; quantitative traits; SNPs.

Cabrera-Bosquet L, Crossa J, von Zitzewitz J, Serret MD, Araus JL (2012) High-throughput phenotyping and genomic selection: The frontiers of crop breeding converge. J. Integr. Plant Biol. 54(5), 312-320.

\section{Introduction}

Grain yield, plant growth and stress adaptation are complex traits controlled by many genes, usually with minor effects and with a high occurrence of epistatic interactions (Li et al. 1997, 2001). This presents limited breeding advances for complex traits. High-throughput phenotyping and genomic selection (GS) of complex traits promise to revolutionize the breeding process by accelerating generation-advance and improving the precision of selection. On one hand, there is the fast, largescale evaluation of plant performance that aims to automatize and standardize the phenotyping process. On the other hand, 
there is the massive use of low-cost genotyping technologies powered by advances in genomic sequencing and the advent of single-nucleotide polymorphism (SNP) markers (Ingvarsson and Street 2011). However, both methodological approaches share in common their empirical nature and their strong dependence on the advances in data gathering and processing (together with the help of robotics). Thus, phenotyping is evolving quickly from the concept of conventional breeding, which relies on the direct measurement of the target trait (e.g. yield) or even from analytical breeding (see Araus et al. 2008), which implies selecting the key secondary trait(s) (i.e. other than the yield or the targeted trait itself), to the remote inference of whole-plant growth, water status or even grain yield using remote sensing approaches. For genotyping, classic marker-assisted selection (MAS) that relies on the identification of quantitative trait loci (QTL) for traits of interest has been found to be far less successful than predicted two decades ago when the target traits for selection were quantitative, such as yield and adaptation to drought or other major abiotic stresses (Maccaferri et al. 2008 and references herein). Phenotyping for quantitative traits has been relatively ignored (even neglected) until recently, when molecular biologists and breeders realized that advances in molecular techniques may only be useful in breeding for quantitative traits if they are based on reliable phenotyping (Araus et al. 2003, 2008). In that context, and taking advantage of the fast development in computing and robotics, a burst of new technological approaches aimed at shifting phenotying from an "art to a technology" has arrived through the development of what are known as high-throughput phenotyping platforms. That includes not just a large set of commercially available instruments that allow this work to be done, but also the emergence of outsourcing services. Thus, following the steps of genomic analyses and markerassisted selection, where outsourcing is a consolidated trend, high-throughput phenotyping has been offered recently on a server provider basis (www.phenofab.com; updated January 2012). The possibility to phenotype thousands of individuals, faster, and with high precision, together with advances and cost reduction in sequencing technologies (Davey et al. 2011), allows GS to be applicable to plant breeding programs. GS promises the power to predict individual genotype adaptability to a specific environment (Crossa et al. 2010; Aguilar et al. 2011; Resende et al. 2012).

In the following discussion, the basics of high-throughput phenotyping and genomic selection are briefly presented together with a few examples.

\section{Genomic Selection: A Step Forward from Marker-assisted Selection}

The traditional approach of MAS, based on bi-parental or association mapping panels, consists of detecting quantitative trait loci (QTL) to further focus on significant regions for assisted selection. Briefly, markers linked to genes are detected, the most favorable alleles and their related effects are determined, and then these are further validated. Nevertheless, MAS seems effective only for major QTL effects (Araus et al. 2008; Maccaferri et al. 2008; von Zitzewitz et al. 2011), and not for complex traits controlled by many genes with small effects, such as grain yield and adaptation to stresses. Further limitations in MAS are the infrequent use of bi-parental populations for germplasm improvement, and the statistical methods used for mapping QTL, which rely on stringent thresholds and single marker analysis models. On the other hand, genomic selection (or genome wide selection) is an approach for improving quantitative (i.e. complex) traits (Meuwissen et al. 2001) that uses all the available molecular markers across the genome and allows calculation of estimated genetic (breeding) values. The following example may illustrate the need for empirical models effective in capturing a large number of minor QTL. In human association genetics, a set of 40 significant markers explained only $5 \%$ of the variability in height while a set of 300000 markers placed simultaneously in a model explained up to $45 \%$ of the heritability for human height (Yang et al. 2010).

GS claims to act in a similar manner and intends to improve the predictions of economically important traits in complex plant and animal breeding programs. Instead of focusing on single marker analysis models with the power to only detect relatively large effects, GS is centralized in a genotypic characterization of several markers (at low cost) to integrate simultaneously (taking into account major and minor effects) in a predictive model with novel statistical methods (de los Campos et al. 2009; Crossa et al. 2010; Vitezica et al. 2011). GS is based on a predictive model that has been trained with a number of individuals ("the training population") that reflects the diversity of the breeding program being evaluated at the phenotypic and genotypic level. As the number of markers increases in the model, the prediction accuracies of genomic estimated breeding values (GEBV) are expected to increase, whereas the single marker effect is expected to decrease in absolute magnitude. Once the model has proven successful, the plant breeder is able to estimate the GEBVs for the next generation cycle through the incorporation of only genomic data. These GEBVs give an ideal criterion for selecting the best performing lines. Figure 1 shows a simple schematic diagram summarizing the implementation of GS in a plant breeding program. However, parametric regression models also provide the opportunity to examine marker effects and study the possible differential response of markers in environments, that is, the gene $\times$ environment interaction effect. In general, Bayesian shrinkage methods do not have an associated test for detecting chromosome regions; however, they can be routinely used for QTL detection. 


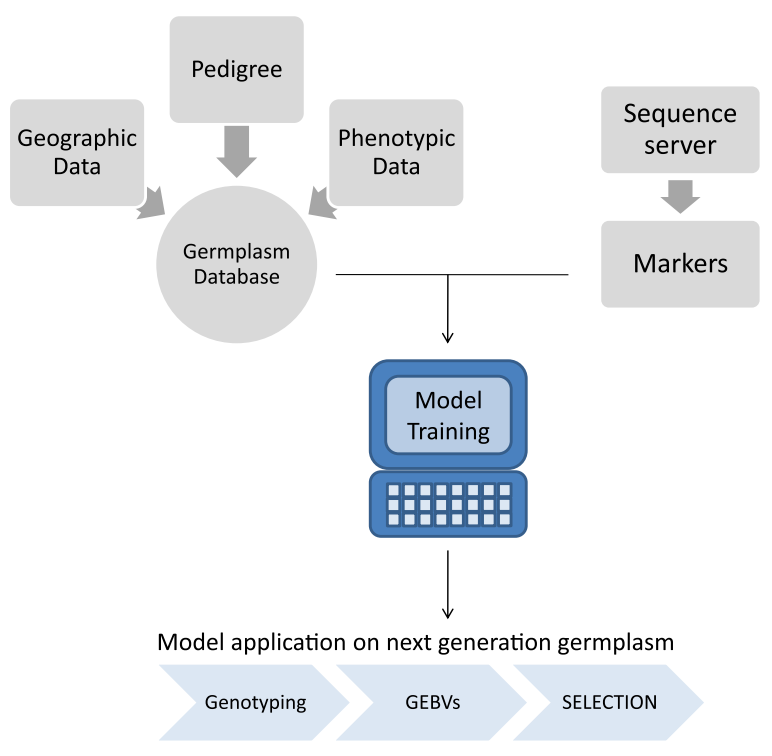

Figure 1. Schematic diagram depicting the steps involved in the use of genomic selection in a plant breeding program.

GEBVs, genomic estimated breeding values.

Although GS was first proposed about 10 years ago (Meuwissen et al. 2001), reports on the use of GS in plants are few and refer mainly to computer simulation studies such as the research of Bernardo and Yu (2007), who concluded that GS was superior to marker-assisted selection in maize. This delay in the application of GS in plant breeding programs can be partly explained by the high costs in genotyping germplasm with a high density of markers at the time. It was not until recently that relatively inexpensive marker technologies have become available as a service platform (i.e. http://www.diversityarrays.com; updated January 2012). Further development of genotyping methods based on direct sequencing of genomic digests have reduced costs tremendously by relying solely on next generation sequencing and non-expensive array-based technologies (Davey et al. 2011; Elshire et al. 2011). The advantage of these methods is that full gain-of-sequence throughput is taken, which is advantageous for polyploid species which cause problems with hybridization, and polymorphism discovery is simultaneous with genotyping. These last methods are one step behind direct sequencing of whole genomes, which will be applicable for GS once prices become affordable.

Diversity Array Technology (DArT) was recently used in studies at the International Maize and Wheat Improvement Center (CIMMYT), where de los Campos et al. (2009), Crossa et al. (2010, 2011), Pérez et al. (2010) and Burgueño et al. (2012) validated GS in plant breeding using genomic regression and showed that models using molecular markers were more accurate in predicting grain yield in wheat and maize than those based on pedigree only. These were the first comprehensive studies demonstrating that genomic selection could be useful in plant breeding. These studies in maize and wheat based on the multi-environment trials of CIMMYT indicated that models for GS can have relatively high predictive ability for genetic values of grain yield and other traits of economic interest under contrasting environmental conditions. GS selection models with pedigree and molecular marker information can be used effectively for selecting individuals whose phenotypes for various traits have yet to be observed under varying environmental conditions, including for example severe water stress. All these studies have indicated that the problem of model choice is population and environment specific and that a 'one-size-fitsall' approach to model choice in GS is not appropriate.

In addition to estimating genetic values, parametric models also provide information on 'marker effects' that can be used to gain a better understanding of the important genomic regions underlying the architecture of traits and genotype $\times$ environment interaction. Principal component analysis of estimated marker effects across environments provides a way of identifying which markers contribute to positive genetic correlations between environments, and which markers have negative responses in other environments and therefore produce interaction with environments. In the following section, an example is examined from the ongoing work of CIMMYT on GS for predicting quantitative traits in plant breeding using dense molecular markers and pedigree.

\section{A Case Study: Patterns of Co-variability of Estimated Marker Effects across Environments for Maize Flowering Genomic Data}

Traits included here were female flowering ( $F F L$ ) (or days to silking), male flowering (MFL) (or days to anthesis), as well as the anthesis-silking interval (ASI) evaluated in 300 maize lines under severe drought stress (SS) and in well-watered (WW) environments (Crossa et al. 2010). The first two component axes of estimated effects of SNP markers in the six trait-environment combinations (MFL-SS, MFL-WW, FFL-SS, FFL-WW, ASI-SS, and $\mathrm{ASI}-\mathrm{WW}$ ) are depicted in Figure 2. The correlation between trait-environment combinations using marker effects and phenotypic data shows that some trait-environment combinations are highly correlated (both phenotypically and genetically). The pattern of correlations between estimated SNP effects reflects the patterns of observed phenotypic correlations. Clearly, the two groups of trait-environment combinations are dominated more by the trait (ASI vs. FFL and MFL) and less by the environment (SS and WW). Phenotypic outcomes and estimates of marker effects for ASI showed relatively small correlations with those of FFL and MFL; this is because ASI is defined as the difference between FFL and MFL, and these two traits are positively correlated. 


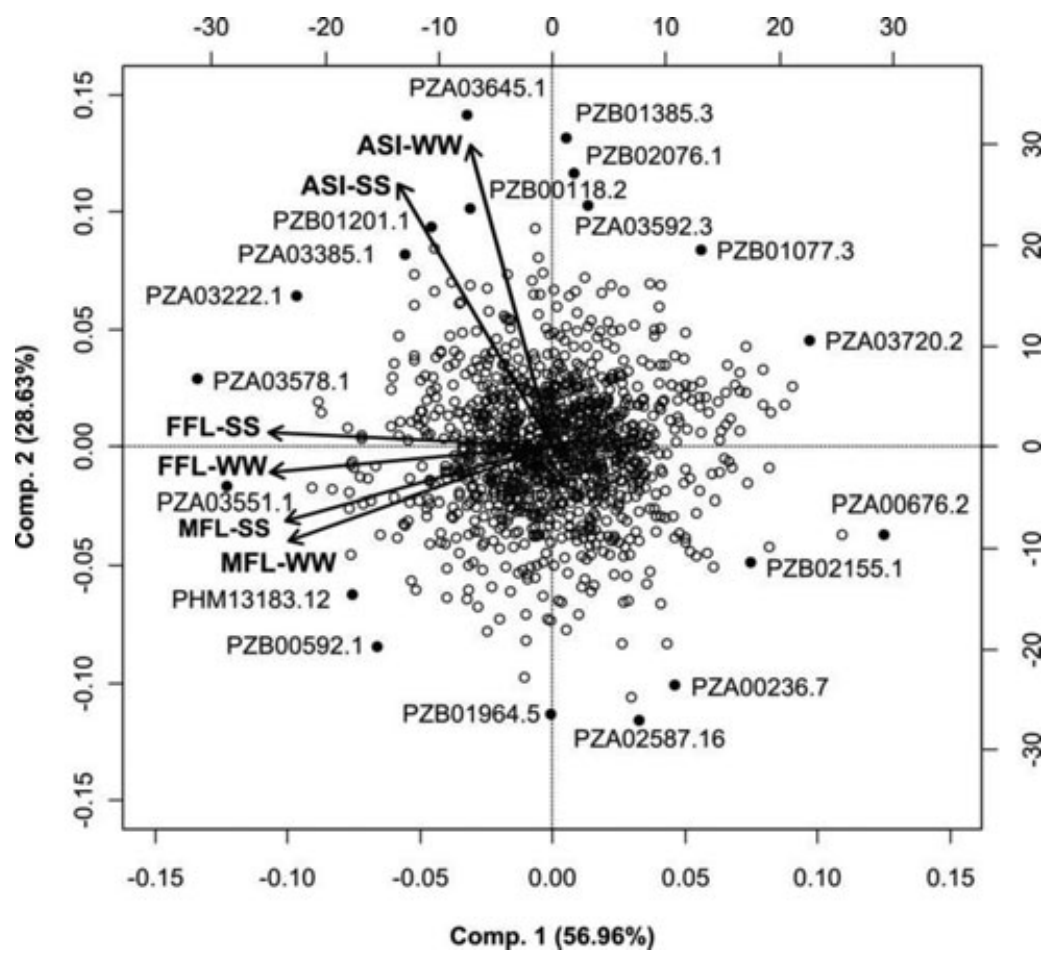

Figure 2. Biplot of the first and second principal component axes of the effects of 1148 SNPs on 3 traits.

Biplot of the first and second principal component axes (Comp. 1 and Comp. 2) of the effects of the 1148 SNPs on maize female flowering (FFL), male flowering (MFL) and anthesis-to-silking interval (ASI) estimated from the full data model M-BL of the maize dataset in each of two environments; severe water stress (SS) and well watered (WW). A total of six trait-environment combinations (FFL-SS, FFL-WW, MFL-SS, MFL-WW, SS-ASI, and WW-ASI) were formed. Only the effects of the 19 SNPs that are located far from the center of the biplot were identified with their corresponding SNP name (filled-in circles) (from Crossa et al. 2010).

Markers with relatively large (in absolute value) estimated effects are identified by name in Figure 2. The marker effects on these traits should be interpreted differently than their effect on grain yield, since the favorable marker allele decreases both female and male flowering times, whereas for ASI, the optimal marker should give an ASI of 0 . The alleles whose estimated effects are located in the left and upper left corner of the biplot (i.e., PZA03551.1, PZA03578.1, PZA03222.1, PZA03385.1, PZB01201.1, and PZB00118.2) increase FFL, MFL, and ASI (they all have positive effects on all trait-environments combinations), whereas those SNPs located on the opposite side of the biplot (lower right corner) (i.e., PZA02587.16, PZA00236.7, PZB0255.1, and PZA00676.2) decrease the value of FFL, MFL, and ASI. Those SNPs whose presence is expected to increase or decrease traits across environments can be viewed as contributing to positive genetic correlations in FFL, MFL, and ASI between environments.

Despite the high heritability (between 0.74 and 0.87 ) found for flowering time and $\mathrm{ASI}$ in this maize trial, results show substantial interaction between molecular marker effects and environment. The biplot in Figure 2 shows SNPs that had very contrasting effects across environments. For example, the minor alleles of SNPs whose estimated effects are located in the upper right corner of the biplot (PZA03592.3, PZB01077.3, and PZB02076.1) increase the anthesis-silking interval under drought and well-watered conditions, but decrease days to male and female flowering. In contrast, the minor alleles of SNPs whose estimated effects are located in the opposite quadrant of the biplot (lower left corner) (PZB00592.1, PHM13183.12, and PZB01964.5) showed a complete rank reversal with respect to the effects of SNPs PZA03592.3, PZB01077.3, and PZB01077.3 on those trait-environment combinations, i.e., a decrease in ASI under SS and WW, and an increase in male and female flowering times. These results are suggestive of an important molecular marker effect $\times$ environment interaction, which in turn causes a genotype $\times$ environment interaction.

\section{Genomic Traits with Genome x Environment}

In a recent comprehensive study, Burgueño et al. (2012) presented multi-environment (multi-trait) models for GS and compared the predictive accuracy of these models 
with: (a) multi-environment analysis without pedigree and marker information, and (b) multi-environment pedigree or/and marker-based models. The authors described a statistical framework for incorporating pedigree and molecular marker information in models for multi-environment data and applied it to data that originated from wheat multi-environment trials. Two prediction problems relevant to plant breeders are considered: predicting the performance of untested genotypes ("newly" developed lines), and predicting the performance of genotypes that have been evaluated in some environments, but not in others. The results confirmed the superiority of models using both marker and pedigree information over those based on pedigree information only. Models with pedigree and/or markers had better predictive accuracy than simple linear mixed models that do not include either of these two sources of information. Burgueño et al. (2012) concluded that the evaluation of such trials can benefit greatly from using multi-environment GS models.

\section{Concluding Remarks on the Plant Genomic Studies}

The results of these studies in CIMMYT are encouraging; they indicate that models for GS can attain high predictive ability for genetic values of traits of economic interest under contrasting environmental conditions. Therefore, GS can be used effectively for selecting individuals whose phenotypes for various traits and in various environments have yet to be observed. As the number of available markers increases (as is expected in the near future), larger gains in predictive ability may be attained.

An advantage of models that include a parametric regression on marker covariates is that, in addition to estimating genetic values, they also provide information on (estimates of) "marker effects". This information can be used to attain a better understanding of the genetic architecture of the traits under study and examine the patterns of response of marker effects across environments. In these studies, separate models were fitted to each trait-environment combination. An alternative to these single-environment models for genomic selection is to use multi-environment (or, equivalently, multi-trait) models where genetic values and marker effects on several traits/environments are jointly estimated. Multi-environment models allow the borrowing of information between correlated environments. Thus it can be speculated that multi-environment genomic models can yield similar or even better predictions for individual environments.

Interestingly, the results of these studies can also be used to generate a better design for field evaluations. For example, they show that prediction of unobserved lines in any of the correlated environments should be relatively accurate, and the scheme for testing these lines in any of those environments should be planned accordingly. It can be speculated that only one of the sets of correlated environments should be included in the trial, because any information lacking for the other environments can be borrowed from the one in use. However, unobserved lines in low correlated environments are expected to be poorly predicted.

\section{High-throughput Phenotyping}

Whereas genomic selection has shown to be useful to predict genetic values of traits of interest, its success in breeding for quantitative traits largely depends on a reliable phenotyping process. In such a way, high-throughput phenotyping platforms (HTPP) could be particularly useful for obtaining detailed measurements of plant characteristics that collectively provide reliable estimates of phenotypic traits (Finkle 2009). These platforms are also useful in modeling for predicting genotypic performance in different climate scenarios. HTPP operations are based on high capacity for data recording/scoring, speed of data collection and processing, and non-destructive, noninvasive and remote sensing phenotyping nature (Prasanna et al. 2012). Whereas HTTPs may help to improve understanding of crop physiology, most powerful techniques for highthroughput phenotyping are empirical (comparable to genomic selection) and founded on a huge capacity for data acquisition and further processing merely based in the need for statistical background but without any need of physiological understanding. We refer to different techniques like near-infrared spectroscopy spectral reflectance, photography and sonar, among others that while already used in the existing HTPPs (mainly developed for plants under controlled conditions), will no doubt be deployed more frequently in future field HTPPs to evaluate complex traits (Montes et al. 2007, 2011). Here we will focus on spectral techniques like near-infrared spectroscopy (NIRS) and plant spectral reflectance which are widely used techniques in plant research that have emerged as promising methods for field-based high-throughput phenotyping of crops subjected to abiotic and biotic stresses (Araus et al. 2002, 2008; Lopes et al. 2011; Prasanna et al. 2012).

\section{Basis of NIRS and Plant Spectral Reflectance}

NIRS is a chemometric technique that combines spectroscopy and mathematics to rapidly produce indirect, quantitative estimates of the concentration of $\mathrm{OH}-, \mathrm{NH}-, \mathrm{CH}$ - or $\mathrm{SH}$ - containing compounds. Owing to the low cost, rapidity, high precision and repeatability of this technique, NIRS has been largely implemented in routine laboratory analyses of grain and fodder quality traits in several crop species such as maize, wheat, sorghum and soybean for assessing several plant traits 


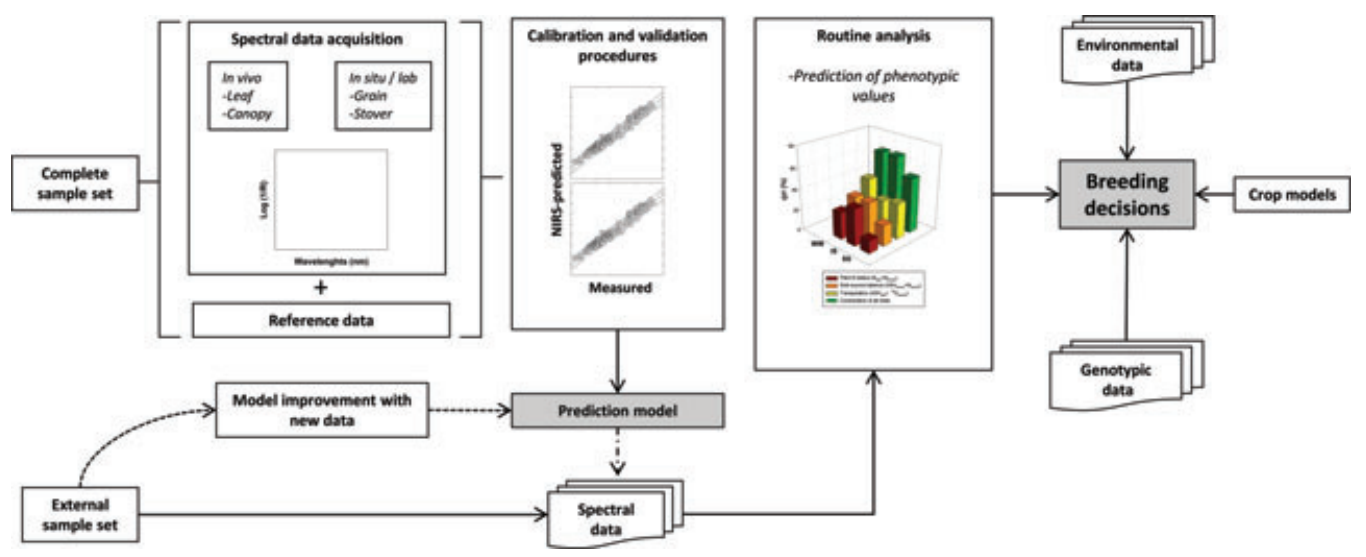

Figure 3. Methodological scheme showing the strategies for the use of spectroradiometrical and near-infrared spectroscopy (NIRS) techniques in plant breeding.

including nitrogen, moisture, fiber, carbohydrates, amino acids, minerals and a number of other plant compounds (see Foley et al. 1998 for a review; Rosales et al. 2011). However, the use of these techniques for indirect assessment of crop growth and yield performance under potential yield and stress conditions has been addressed more recently. In fact NIRS is not just limited to analyzing any specific compound or metabolite relevant for adaptation to abiotic stresses such the stable isotope signatures of carbon (Ferrio et al. 2001) and oxygen (Cabrera-Bosquet et al. 2011a) in a fast, albeit non-destructive and comparatively inexpensive manner; NIRS measurement of kernels has also been used to infer genotypic variability in wheat yield (Ferrio et al. 2004).

Plant spectral reflectance or hyperspectral reflectance spectroscopy is based on the different pattern of light reflectance on leaves at different wavelengths through the photosynthetically active radiation (PAR, 400-700 $\mathrm{nm}$ ), near infrared radiation (NIR, 700-1 $200 \mathrm{~nm}$ ) and shortwave infrared (up to $2500 \mathrm{~nm}$ ) regions of the electromagnetic spectrum. Spectral reflectance information from leaves or canopies is used to build vegetation indices (VIs), which are simple operations (e.g., ratios and differences) between spectral reflectance data at given wavelengths. Vegetation indices are related to different plant characteristics such as photosynthetic active biomass, pigment content and water status (see Araus et al. 2001, 2002; Ollinger 2008 for a review). Thus vegetation indices have been used to predict green biomass, leaf area, chlorophyll content and yield in wheat and maize under field conditions (Aparicio et al. 2000; Araus et al. 2001; Babar et al. 2006; Marti et al. 2007; Cabrera-Bosquet et al. 2011b). Other VIs such as the photochemical reflectance index (PRI) and the water index (WI) may allow inference in photosynthetic efficiency and plant water status (Araus et al. 2001, 2002; Babar et al. 2006).

Both NIRS spectroscopy and plant spectral reflectance techniques rely on the development of calibration models relating spectral information and reference data (i.e. traditional laboratory analyses) of the trait. Usually, a sub-sample of a complete data set representing the entire population in terms of range of spectral variation is used for calibration development using the appropriate mathematical treatments and algorithms to build robust prediction models (Shenk and Westerhaus 1993; Montes et al. 2007). Once calibration models have been successfully validated, they can be later employed in routine analyses to predict phenotypic values on external data sets using spectral data and further used in combination with environmental and genotypic data to make breeding decisions (Figure 3). Nevertheless, in the case of laboratory-based NIRS, canopy spectral reflectance data has also been proposed for measuring complex traits such as grain yield for crops like durum wheat (Ferrio et al. 2005) and maize (Weber et al. 2012). In that case, instead of formulating indices, the approach is similar to NIRS in the sense that whole spectra are used in an empirical basis (without aprioristic assumptions taken for VI) to fit the best model predicting the trait of interest. In fact, hyperspectral field spectroradiometers may be comparable to a portable NIRS in terms of range and density of wavelengths measured, especially if they are equipped with their own light source (i.e. active sensors), which largely reduces the difficulties associated with changes in solar radiation and the angle experienced during measurements (Weber et al. 2012).

\section{NIRS and Plant Spectral Reflectance when Phenotyping for Complex Traits}

Spectroradiometric techniques can be efficiently used in breeding programs where thousands of individuals must be screened. For example, spectral reflectance may provide a fast and non-destructive evaluation of dynamic traits in $\mathrm{C}_{3}$ and $\mathrm{C}_{4}$ crops such as biomass accumulation (Babar et al. 2006), radiation use efficiency (Jørgensen et al. 2002) and yield (Ferrio et al. 2005). For instance, predictions of yield and other plant traits at early stages (e.g. before flowering) 
may speed-up design of test crosses at anthesis, saving time and costs (Weber et al. 2012). NIRS can be very useful as a fast, cost-effective and non-destructive method for screening genotypes in breeding nurseries or in the initial stages of breeding programs where seed availability is a major constraint. In recent work, Cabrera-Bosquet et al. (2011a) used NIRS to accurately predict genotypic differences in kernel and leaf ash content and nitrogen in maize grown under different water treatments (Figure 3). Using the same rationale as for spectral reflectance, NIRS has also been proposed to predict traits related to genotypic adaptation to water stress such as the stable isotope composition of carbon (Ferrio et al. 2001) and oxygen (Cabrera-Bosquet et al. 2011a) in mature kernels. Besides phenotyping, these techniques and particularly the evaluation of $\mathrm{VI}$ are very promising for a fast non-destructive evaluation of soil heterogeneity (Masuka et al. 2012; Prasanna et al. 2012) as well as for crop management related to conservation agriculture, for example see Verhulst and Govaerts (2010).

Although the use of NIRS and spectral reflectance techniques as a high-throughput phenotyping tool is very promising, its application is in its infancy and requires further technical and logistic advances. Technical solutions have been proposed, like the use of NIRS on agricultural harvesters and canopy spectroradiometers mounted on tractors (Montes et al. 2007, 2011), decreasing the manpower and costs and making the acquisition of spectral information in a large number of plots much faster and more precise. Aerial platforms are also very suited for field phenotyping. These include different options from remote controlled helicopters and 'policopters', airplanes to balloons or cranes and conveyers.

Conversely, one limitation of the application of these techniques in high-throughput phenotyping is handling the extensive data produced. This may be extended to other of the more successful phenotyping approaches, such fully-automated digital imaging systems that precisely measure the growth of a crop plant throughout its lifecycle using 3D color digital imaging, which is one of the key developments of the current HTPPs designed for potted plants (Golzarian et al. 2011; Hartmann et al. 2011).

Manipulation and analyses of huge datasets generated during the phenotyping of thousands of individuals largely benefits from the availability of robust databases and novel statistical and mathematical tools. A suitable database must essentially allow storage, visualization and analysis of phenotypic data, but it must be also able to hold associated metadata such as genetic, molecular and metabolic profiling information, with appropriate tools for genetic analyses and association with phenotypic values. In addition, a precise characterization of environmental and growing conditions is crucial for the interpretation and analysis of the $\mathrm{G} \times \mathrm{E}$ interaction of complex traits (Chenu et al. 2009). The development of mathematical and statistical methods is also necessary for the analysis and inter- pretation of complex dynamic multi-temporal and multi-scale phenotypic characters such as biomass accumulation, developmental processes and temperature-dependent traits (Yin et al. 2004). Finally, an adequate structure to the database including the use of standards, comprehensive metadata description and plant ontologies enables interconnection with other available biological databases and resources (Ilic et al. 2007; Furbank and Tester 2011; http://www. plantontology.org/). Several initiatives such as the PHENOPSIS DB (Fabre et al. 2011), Xeml Lab (Hannemann et al. 2009) and PODD (http://www.plantphenomics.org.au/PODDProject) are publicly available, and these contain phenotypic data and experimental and environmental metadata generated by phenotyping platforms and multi-laboratory experiments.

\section{The Way Ahead}

The accurate prediction of complex traits will highly depend on the quality of the phenotypic data collected. Genotyping by direct sequencing is already affordable for GS (http://www.maizegenetics.net/gbs-overview; updated January 2012) and can only be expected to become more affordable and more informative. GS suggests substantial progress in the efficiency of selection combining field characterization and selection with the addition of thousands of markers simultaneously in a prediction model (Meuwissen et al. 2001). There is already evidence in plant species with model predictions showing a high efficiency of selection compared with phenotypic selection, including characteristics of complex inheritance (Crossa et al. 2010; Heffner et al. 2011; Resende et al. 2011). This challenges the paradigms that sustain the current designs in plant breeding programs.

Phenotyping still remains the weak point in the breeding process. High-throughput phenotyping platforms are in their infancy, and will succeed when high-throughput platforms for field conditions become reliable in accuracy, in capacity, and become affordable. Phenotyping technologies need to tackle selected traits and methodologies, and also the spatial and temporal variability inherent in field testing (Masuka et al. 2012; Prasanna et al. 2012). Moreover, the future also implies using more sophisticated crop models, which will allow the deciphering and even the designing of traits involved in the genetic variability for quantitative traits (Yin et al. 2004; Chenu et al. 2009). In addition, the models used for the integration of complex gene-phenotype relationships in breeding are becoming increasingly effective (Messina et al. 2011). Physiological understanding will remain a cornerstone for phenotyping, and by default, for breeding. Moreover, linking both the phenotypic and the genotypic boundaries of the breeding process will rely more heavily than in the past on data analysis. 


\section{Acknowledgments}

Participation of José Luis Araus and María Dolors Serret was supported by the Spanish Project AGL2010-20180 (subprogram AGR) and the FP7 European Project OPTICHINA (266045).

Received 22 Jan. 2012 Accepted 14 Feb. 2012

\section{References}

Aguilar I, Misztal I, Tsuruta S, Wiggans GR, Lawlor TJ (2011) Multiple trait genomic evaluation of conception rate in Holsteins. J. Dairy Sci. 94, 2621-2624.

Aparicio N, Villegas D, Casadesus J, Araus JL, Royo C (2000) Spectral reflectance indices for assessing durum wheat biomass, green area, and yield under Mediterranean conditions. Agron. J. 92, 83-91.

Araus JL, Casadesús J, Bort J (2001) Recent tools for the screening of physiological traits determining yield. Chapter 5. In: Reynolds M, Ortiz-Monasterio I, McNab A, eds. Application of Physiology in Wheat Breeding. CIMMYT, Mexico, D.F. pp: 59-77.

Araus JL, Slafer GA, Reynolds MP, Royo C (2002) Plant breeding and water stress in $\mathrm{C}_{3}$ cereals: What to breed for? Ann. Bot. 89, 925-940.

Araus JL, Bort J, Steduto P, Villegas D, Royo C. (2003) Breeding cereals for Mediterranean conditions: Ecophysiological clues for biotechnology application. Ann. Appl. Biol. 142, 129-141.

Araus JL, Slafer GA, Royo C, Serret MD (2008) Breeding for yield potential and stress adaptation in cereals. Crit. Rev. Plant Sci. 27, $1-36$.

Babar MA, van Ginkel M, Klatt AR, Prasad B, Reynolds MP (2006) The potential of using spectral reflectance indices to estimate yield in wheat grown under reduced irrigation. Euphytica 150, 155-172.

Bernardo R, Yu J (2007) Prospects for genome-wide selection for quantitative traits in maize. Crop Sci. 47, 1082-1090.

Burgueño J, de los Campos G, Weigel K, Crossa J (2012) Genomic prediction of breeding values when modeling genotype $\times$ environment interaction using pedigree and dense molecular markers. Crop Sci. doi: 10.2135/cropsci2011.06.0299.x

Cabrera-Bosquet L, Sánchez C, Rosales A, Palacios-Rojas N, Araus JL (2011a) NIRS-assessment of $\delta^{18} \mathrm{O}$, nitrogen and ash content for improved yield potential and drought adaptation in maize. J. Agric. Food Chem. 59, 467-474.

Cabrera-Bosquet L, Molero G, Stellacci AM, Bort J, Nogués S, Araus JL (2011b) NDVI as a potential tool for predicting biomass, nitrogen content and growth in potted wheat genotypes. Cereal Res. Comm. 39, 147-159.

Chenu K, Chapman SC, Tardieu F, McLean G, Welcker C, Hammer GL (2009) Simulating the yield impacts of organ-level quantitative trait loci associated with drought response in maize: A 'Gene-toPhenotype' modeling approach. Genetics 183, 1507-1523.

Crossa J, de los Campos G, Pérez P, Gianola D, Dreisigacker S, Burgueño J, Araus JL, Makumbi D, Yan J, Singh R, Arief V, Banziger M, Braun HJ (2010) Prediction of genetic values of quantitative traits in plant breeding using pedigree and molecular markers. Genetics 186, 1-12.

Crossa J, Pérez P, de los Campos G, Mahuku G, Dreisigacker S, Magorokosho C (2011) Genomic selection and prediction in plant breeding. J. Crop Improv. 25, 239-261.

Davey JW, Hohenlohe PA, Etter PD, Boone JQ, Catchen JM, Blaxter ML (2011) Genome-wide genetic marker discovery and genotyping using next-generation sequencing. Nat. Rev. Genet. 12, 499-510.

de los Campos G, Naya H, Gianola D, Crossa J, Legarra A, Manfredi E, Weigel K, Cotes JM (2009) Predicting quantitative traits with regression models for dense molecular markers and pedigree. Genetics 182, 375-385.

Elshire RJ, Glaubitz JC, Sun Q, Poland JA, Kawamoto K, Buckler ES, Mitchell SE (2011) A robust, simple genotyping-by-sequencing (GBS) approach for high diversity species. PLOS ONE 6, e19379.

Fabre J, Dauzat M, Nègre V, Wuyts N, Tireau A, Gennari E, Neveau P, Tisné S, Massonet C, Hummel I, Granier C (2011) PHENOPSIS DB: An information system for Arabidopsis thaliana phenotypic data in an environmental context. BMC Plant Biol. 11, 77-84.

Ferrio JP, Bertran E, Nachit MM, Royo C, Araus JL (2001) Near infrared reflectance spectroscopy as a new surrogate analysis for $\Delta^{13} \mathrm{C}$ in mature kernels of durum wheat. Aust. J. Agric. Res. 52, 809-816.

Ferrio JP, Bertran E, Nachit MM, Català J, Araus JL (2004) Estimation of grain yield by near-infrared reflectance spectroscopy in durum wheat. Euphytica 137, 373-380.

Ferrio JP, Villegas D, Zarco J, Aparicio N, Araus JL, Royo C (2005) Assessment of durum wheat yield using visible and near-infrared reflectance spectra of canopies. Field Crops Res. 94, 126-148.

Finkle E (2009) With "phenomics" plant scientists hope to shift breeding into overdrive. Science 325, 380-381.

Foley WJ, Mcllwee A, Lawler I, Aragones L, Woolnough AP, Berding $\mathbf{N}$ (1998) Ecological applications of near infrared reflectance spectroscopy - a tool for rapid, cost-effective prediction of the composition of plant and animal tissues and aspects of animal performance. Oecologia 116, 293-305.

Furbank RT, Tester M (2011) Phenomics - technologies to relieve the phenotyping bottleneck. Trends Plant Sci. 16, 635-644.

Golzarian MR, Frick RA, Rajendran K, Berger B, Roy S, Tester M, Lun DS (2011) Accurate inference of shoot biomass from highthroughput images of cereal plants. Plant Methods 7, 2.

Hannemann J, Poorter H, Usadel B, Bläsing OE, Finck A, Tardieu F, Atkin OK, Pons T, Stitt M, Gibon Y (2009) Xeml Lab: A tool that supports the design of experiments at a graphical interface and generates computer-readable metadata files, which capture information 
about genotypes, growth conditions, environmental perturbations and sampling strategy. Plant Cell Environ. 32, 1185-200.

Hartmann A, Czauderna T, Hoffmann R, Stein N, Schreiber F (2011) HTPheno: An image analysis pipeline for high-throughput plant phenotyping. BMC Bioinf. 12, 148.

Heffner EL, Jannink JL, Sorrells ME (2011) Genomic selection accuracy using multifamily prediction models in a wheat breeding program. Plant Genome 4, 65-75.

llic K, Kellogg EA, Jaiswal P, Zapata F, Stevens PF, Vincent LP, Avraham S, Reiser L, Pujar A, Sachs MM, Whitman NT, McCouch SR, Schaeffer ML, Ware DH, Stein LD, Rhee SY (2007) The plant structure ontology, a unified vocabulary of anatomy and morphology of a flowering plant. Plant Physiol. 143, 587-599.

Ingvarsson PK, Street NR (2011) Association genetics of complex traits in plants. New Phytol. 189, 909-922.

Jørgensen U, Mortensen J, Ohlsson C (2002) Light interception and dry matter conversion efficiency of miscanthus genotypes estimated from spectral reflectance measurements. New Phytol. 157, 263270.

Li ZK, Pinson SR, Park WD, Paterson AH, Stansel JW (1997) Epistasis for three grain yield components in rice (Oryza sativa L.). Genetics 145, 453-465.

Li ZK, Luo JL, Mei HW, Wang DL, Shu QY, Tabien R, Zhong DB, Ying CS, Stansel JW, Khush GS, Paterson AH (2001) Overdominant epistatic loci are the primary genetic basis for inbreeding depression and heterosis in rice. I. Biomass and grain yield. Genetics 158, 1737-1753.

Lopes MS, Araus JL, Van Heerden PDR, Foyer CH (2011) Enhancing drought tolerance in $\mathrm{C}_{4}$ crops. J. Exp. Bot. 62, 3135-3153.

Maccaferri M, Sanguineti MC, Corneti S, Araus JL, Ben Salem M, Bort J, DeAmbrogio E, Garcia del Moral L, Demontis A, ElAhmed A, Elouafi I, Maalouf F, Machlab H, Martos V, Nachit MN, Nserallah N, Ouabbou H, Royo C, Slama A, Villegas D, Tuberosa R (2008) Quantitative trait loci for grain yield and adaptation of durum wheat (Triticum durum Desf.) across a wide range of water availability. Genetics 178, 489-511.

Marti J, Bort J, Slafer G, Araus JL (2007) Can wheat yield be assessed by early measurements of NDVI? Ann. Appl. Biol. 150, 253-257.

Masuka B, Araus JL, Das B, Sonder K, Cairns JE (2012) Phenotyping for abiotic stress tolerance in maize. J. Integr. Plant Biol. 54(4), 238249.

Messina CD, Podlich D, Dong Z, Samples M, Cooper M (2011) Yieldtrait performance landscapes: From theory to application in breeding maize for drought tolerance. J. Exp. Bot. 62, 855-868.

Meuwissen THE, Hayes BJ, Goddard ME (2001) Prediction of total genetic values using genome-wide dense marker maps. Genetics 157, 1819-1829.

Montes J, Melchinger A, Reif J (2007) Novel throughput phenotyping platforms in plant genetic studies. Trends Plant Sci. 12, 433-436.
Montes JM, Technow F, Dhillon BS, Mauch F, Melchinger AE (2011) High-throughput non-destructive biomass determination during early plant development in maize under field conditions. Field Crops Res. 121, 268-273.

Ollinger SV (2008) Sources of variability in canopy reflectance and the convergent properties of plants. New Phytol. 189, 375-394.

Pérez P, de Los Campos G, Crossa J, Gianola D (2010) Genomicenabled prediction based on molecular markers and pedigree using the Bayesian linear regression package in R. Plant Gen. 3, 106-116.

Prasanna BM, Araus JL, Crossa J, Cairns JE, Palacios N, Mahuku G, Das B, Magorokosho C (2012) High-throughput and precision phenotyping in cereal breeding programs. In: Cereal Genomics, 2nd Edition, Springer (in press).

Resende MFR, Muñoz P, Acosta JJ, Peter GF, Davis JM, Grattapaglia D, Resende MDV, Kirst M (2012) Accelerating the domestication of trees using genomic selection: Accuracy of prediction models across ages and environments. New Phytol. 193, 617624.

Rosales A, Galicia L, Oviedo E, Islas C, Palacios-Rojas N (2011) Near-infrared reflectance spectroscopy (NIRS) for protein, tryptophan, and lysine evaluation in quality protein maize (QPM) breeding programs. J. Agric. Food Chem. 59, 10781-10786.

Shenk JS, Westerhaus MO (1993) Near infrared reflectance analysis with single and multiproduct calibrations. Crop Sci. 33, 582-584.

Verhulst N, Goaverts B (2010) The normalized difference vegetation index (NDVI) GreenSeeker ${ }^{\mathrm{TM}}$. Handheld sensor: Toward the integrated evaluation of crop management. Part A: Concepts and case studies. CIMMYT, Mexico, D.F.

Vitezica ZG, Aguilar I, Misztal I, Legarra A (2011) Bias in genomic predictions for populations under selection. Genet. Res. 93, 357366.

von Zitzewitz J, Cuesta-Marcos A, Condon F, Castro AJ, Chao S, Corey A, Filichkin T, Fisk SP, Gutierrez L, Haggard K, Karsai I, Muehlbauer GJ, Smith KP, Veisz O, Hayes PM (2011) The genetics of winterhardiness in barley: Perspectives from genomewide association mapping. Plant Gen. 4, 76-91.

Yang J, Benyamin B, McEvoy BP, Gordon S, Henders AK, Nyholt DR, Madden PA, Heath AC, Martin NG, Montgomery GW, Goddard ME, Visscher PM (2010) Common SNPs explain a large proportion of the heritability for human height. Nat. Gen. 42, 565569.

Yin X, Struick PC, Kropff MJ (2004) Role of crop physiology in predicting gene-to-phenotype relationships. Trends Plant Sci. 9, 426-432.

Weber VS, Araus JL, Cairns JE, Sanchez C, Melchinger AE, Orsini E (2012) Prediction of grain yield using reflectance spectra of canopy and leaves in maize plants grown under different water regimes. Field Crops Res. (in press). 